\title{
Evaluation of the Efficacy of Sorafenib on Overall Survival in Patients with Hepatocellular Carcinoma using FT Rate: A Devised Index
}

\author{
Takamasa Ohki (D) Mayuko Kondo · Yuki Karasawa · Satoshi Kawamura • \\ Shuuya Maeshima · Kentaro Kojima • Michiharu Seki • \\ Nobuo Toda $\cdot$ Yoshinobu Shioda $\cdot$ Kazumi Tagawa
}

Received: February 9, 2017 / Published online: April 7, 2017

(C) The Author(s) 2017. This article is an open access publication

\begin{abstract}
Introduction: Transcatheter arterial chemoembolization (TACE) is the first-line treatment for intermediate stage hepatocellular carcinoma (HCC) and prolongs survival in HCC patients. However, repeated TACE results in diminished therapeutic response. In addition, the superiority of sorafenib to TACE monotherapy or combined therapy in patients with HCC is still controversial. The prognosis of HCC has many variables and, thus, the effect of a specific treatment is difficult to evaluate. The frequency of treatments per year (FT rate) used in this study was obtained by dividing the total number of radiofrequency ablations and TACE or transcatheter arterial infusion treatments by the years of survival. The aim of this study was to evaluate the overall survival (OS) of TACE versus sorafenib using the FT rate.
\end{abstract}

Enhanced content To view enhanced content for this article go to http://www.medengine.com/Redeem/ 7B08F06008CF004B.

T. Ohki $(\bowtie) \cdot$ M. Kondo · Y. Karasawa .

S. Kawamura - S. Maeshima - K. Kojima · M. Seki ·

N. Toda

Department of Gastroenterology, Mitsui Memorial

Hospital, Tokyo, Japan

e-mail: lily.rover220@gmail.com

Y. Shioda $\cdot$ K. Tagawa

Department of Surgery, Shioda Hospital, Katsuura

City, Japan
Methods: We compared the OS of patients with recurrence of HCC receiving repeated TACE monotherapy $(\mathrm{CON})$ with those receiving therapy switched from TACE to sorafenib (SOR). In addition, a one-to-one FT rate matching cohort consisting of matched SOR (mSOR) and matched CON (mCON) was determined using the propensity score matching method, and OS in the cohort was evaluated. Factors influencing survival were evaluated using Cox proportional hazard regression analysis in all patients and the FT rate matched cohort.

Results: In the FT rate matched cohort, the cumulative survival rate was significantly higher in the mSOR group compared with the mCON group. Multivariate regression analysis of the FT rate matched cohort showed the FT rate and sorafenib to be significant variables for survival with a hazard ratio (HR) of 2.86 $(p<0.001)$ and $0.42(p=0.008)$, respectively.

Conclusion: Early switching from TACE to sorafenib therapy may prolong OS in HCC patients unresponsive to TACE. The present study indicates that the FT rate is potentially a useful index in evaluating the outcome for patients at various stages and treatment regimens.

Funding: Bayer Yakuhin, Ltd.

Keywords: Frequency of treatments per year; HCC; Hepatocellular carcinoma; Oncology; Overall survival; Sorafenib; TACE; Transcatheter arterial chemoembolization 


\section{INTRODUCTION}

Hepatocellular carcinoma (HCC) accounts for $7.5 \%$ of all cancers and is the second most common cause of cancer-related death worldwide [1]. There is a lack of effective therapy for the treatment of advanced HCC and many cases have a poor prognosis. Although there is an initial beneficial response in patients receiving surgery, the recurrence rate of HCC after surgery ranges from $60 \%$ to $90 \%$ over 5 years [2-4]. Transcatheter arterial chemoembolization (TACE) is the first-line treatment for intermediate stage HCC patients with recurrence or unresectable HCC [5]. TACE prevents recurrence of HCC for up to 6 months after surgery [6], and shows improved survival [7, 8]. However, with repeated TACE, therapeutic response often is diminished [9].

Sorafenib, a molecular-targeted drug, inhibits the Raf/MEK/ERK signaling pathway that controls tumor cell proliferation and inhibits neoangiogenesis by blocking VEGF receptors (VEGFRs) [10]. Phase III randomized controlled trials showed that patients with advanced HCC who received sorafenib had significantly prolonged overall survival (OS) compared with those who received placebo $[11,12]$.

The effects of therapeutic strategies including repeated TACE monotherapy, TACE and sorafenib combination therapy, sorafenib maintenance therapy after TACE, and switching therapy from TACE to sorafenib after TACE refractory in patients with HCC have been widely reported; however, the results remain controversial [13-16]. In addition, a number of meta-analyses of TACE and sorafenib combination treatment have shown conflicting data [17-22], indicating the difficulty of evaluating HCC treatment.

We previously reported that the therapeutic strategy of early switching to sorafenib with TACE as a maintenance therapy prolonged progression-free survival (PFS) compared with repeated conventional TACE monotherapy in HCC patients unresponsive to TACE [23, 24]. In the present study, we employed an index obtained by dividing the total number of radiofrequency ablation (RFA), TACE, and transcatheter arterial infusion (TAI) procedures by the years of survival to evaluate the efficacy of repeated TACE monotherapy and early switching from TACE to sorafenib therapy on OS.

\section{METHODS}

\section{Patients}

Patients treated with curative radiofrequency ablation (RFA) against primary HCC from January 2000 to December 2015 were selected and retrospectively evaluated. Sorafenib was approved for the indication of HCC in 2008 in Japan. This study aimed to evaluate the effect of the change in the therapeutic strategy for HCC before and after approval of sorafenib in Japan. Intermediate/advanced stage HCC patients whose treatment had been switched from TACE to sorafenib with maintenance therapy of TACE after being found unresponsive to TACE were designated the sorafenib (SOR) group; those treated with repeated TACE monotherapy alone were designated the control (CON) group. We compared the OS of the SOR group with that of the CON group. The diagnosis of HCC was based on typical findings from imaging such as arterial hyperattenuation and portal hypoattenuation from dynamic contrast-enhanced CT or gadolinium-ethoxybenzyl-diethylenetriamine pentaacetic acid (Gd-EOB-DTPA) MRI.

All procedures followed were in accordance with the ethical standards of the responsible committee on human experimentation (institutional and national) and with the 2013 revised Helsinki Declaration of 1964. Informed consent was obtained from all patients included in the study.

\section{Methods}

\section{FT Rate and Survival}

The frequency of treatments per year (FT rate) was determined by dividing the total number of RFA and TACE/TAI procedures undergone by the years of survival. To evaluate the relationship between FT rate and survival, the CON 
group was divided into four subgroups according to FT rate as follows: group A, $<0.5$; group $\mathrm{B}$, $\geq 0.5-<1.0$; group $\mathrm{C}, \geq 1.0-<2.0$; and group $\mathrm{D}$, $\geq 2$.0. Cumulative survival rates were estimated for every subgroup using the Kaplan-Meier method. To evaluate factors influencing survival other than sorafenib, univariate and multivariate Cox proportional hazard regression analyses were conducted in the CON group. The following were considered to be independent variables in the hazard model: age, gender, number of RFA, TACE, and TAI procedures undergone, HCV-Ab, albumin, total bilirubin, ALT, PT activity, platelet count, alpha-fetoprotein (AFP), des- $\gamma$-carboxy prothrombin (DCP), tumor count, tumor diameter, and FT rate.

\section{Effect of Sorafenib on Survival}

To evaluate the effect of sorafenib on survival, we used propensity scoring to extract an FT rate matched cohort. The propensity score was calculated using the FT rate, and the FT rate matched one-to-one cohorts consisting of SOR patients (mSOR group) and CON patients (mCON group) were determined. The cumulative survival rate was evaluated using the Kaplan-Meier method, and 5- and 10-year survival rates were compared for both SOR vs. CON and mSOR vs. mCON. Factors influencing survival were evaluated using univariate and multivariate Cox proportional hazard regression analysis of the FT rate matched cohorts. Univariate analysis was performed using the following independent variables: age, gender, sorafenib administration, FT rate, tumor count, tumor diameter, albumin, total bilirubin, PT activity, AFP, and platelet count. Sorafenib was administered to TACE-unresponsive patients diagnosed with progressive disease (PD) twice in a row 1 or 2 months after TACE. The starting dose of sorafenib was $400 \mathrm{mg} /$ day and, in principle, the daily dose was increased up to $800 \mathrm{mg} /$ day according to patient acceptability. In the SOR group, TACE or TAI was performed as a maintenance therapy according to response of tumor. PD was defined according to the modified response evaluation criteria in solid tumors (mRECIST) [25].

\section{Patient Background and Treatment}

Patient background information included the following: age, gender, median OS, number of $\mathrm{RFA} / \mathrm{TACE} / \mathrm{TAI}$ procedures, HCV-Ab, excessive alcohol intake, Child-Pugh classification, albumin, total bilirubin, ALT, PT activity, platelet count, AFP, DCP, tumor count, and tumor diameter. For the SOR group, dose, treatment duration period, and adverse events (AEs) were collected. A dose of sorafenib that was continued for more than 1 month was defined as the maintenance dose. AEs were determined according to Common Terminology Criteria for Adverse Events (CTCAEs) version 4.0. TACE procedure had been performed using the Seldinger technique with specific procedures. After routine preoperative preparation, a 5-Fr catheter was inserted into the celiac trunk, hepatic artery, or superior mesenteric artery through the femoral artery on the basis of the tumor arterial blood supply confirmed by arteriography. With fluoroscopy guidance, the tip of the catheter was inserted into the super-selected tumor-feeding branches with the help of a guidewire. After the position of the catheter was confirmed by fluoroscopy, the target vessel was embolized with an emulsion of mixed mitomycin (2-10 mg) and doxorubicin (10-40 mg) with lipiodol $(2-20 \mathrm{~mL})$. If necessary, a gelatin sponge or polyvinyl alcohol particles were injected to embolize tumor-feeding arterioles.

\section{Statistics}

Descriptive statistics were expressed as percentage, median (interquartile range), and mean \pm SD. Factors influencing survival were evaluated using Cox proportional hazard model, and multivariate analysis was performed using significant variables in the univariate analysis. Survival was censored at December 31, 2015. The cumulative survival rate was estimated using the Kaplan-Meier method of comparing values with a log-rank test. Five- and 10-year survival rates were estimated using a log-normal distributed regression model applied to the probability distribution of OS. ANOVA, Chi square test, 
and Fisher's exact test were applied to compare variables between groups. Alpha was set at 0.05 ; all $p$ values were two-sided. To reduce the influence of bias, propensity score matching was utilized to adjust the differences between groups. The propensity score was estimated by employing a logistic regression model with exposure or not to sorafenib as the dependent variable, and the FT rate was defined as an independent variable. A nearest available neighbor-matched analysis on the basis of the estimated propensity score of each patient was one-to-one matched. Statistics were generated using StatView, version 5.0 and JMP, version 11.0 .

\section{RESULTS}

\section{Patient Characteristics}

Baseline patient characteristics in the SOR group, the CON group, and the CON subgroups are shown in Table 1 . The SOR group showed a lower median age and more RFA/ TACE/TAI procedures than the CON group. Among the subgroups, significant differences were found in median OS, numbers of RFA/ TACE/TAI procedures, proportions of Child-Pugh class B or C, albumin, total bilirubin, PT activity, platelet count, and tumor count. Among the subgroups, median OS decreased as FT rate increased. The SOR group showed relatively longer median OS for their FT rate.

\section{FT Rate and OS}

Significant differences were found in the cumulative survival rates among subgroups A to D (Fig. 1). Among the subgroups, survival curves showed that the cumulative survival rate decreased as the FT rate increased. Table 2 shows factors influencing survival in the CON group. The multivariate analysis identified the number of RFAs (HR 0.56, $p<0.01$ ), TACEs/ TAIs (HR $0.85, p<0.01$ ), and an FT rate of $\geq 1.0$ (HR 30.3, $p<0.01$ ) as influential variables for OS.

\section{Sorafenib and OS}

Table 3 shows patient characteristics of the FT rate matched cohort. Significant differences were found in gender, number of RFA procedures, number of RFA/TACE/TAI procedures, albumin, and AFP between the $\mathrm{mSOR}$ and mCON groups. There were more RFA and RFA/ TACE/TAI procedures in mSOR compared with the mCON group. Figure 2 shows the cumulative survival rates for all patients and the FT rate matched cohort. No significant differences were found in the cumulative survival rate between the SOR and CON groups $(p=0.71)$ (Fig. 2a). However, there were significant differences in the cumulative survival rates between the mSOR and mCON groups ( $p=0.005)$ (Fig. $2 b)$. The estimated 5-year survival rates were $57.6 \%$ and $28.8 \%$ in the $\mathrm{mSOR}$ and $\mathrm{mCON}$ groups, respectively, and 10-year survival rates were $19.0 \%$ and $5.2 \%$, respectively. Table 4 shows the factors influencing OS in the FT rate matched cohort. As a result of univariate analysis, gender, age, sorafenib, FT rate, and platelet count were found to be significant variables. Multivariate analysis identified that sorafenib (HR $0.42, p=0.008)$ and FT rate (HR 2.86, $p<0.001)$ were significantly associated with OS.

\section{Sorafenib Dose Modification and AEs}

Median (interquartile range) and minimum and maximum administration periods of sorafenib were 373 (83-656) and 10 and 1752 days, respectively. Percentage and number of patients according to maintenance daily dose of sorafenib were as follows: $200 \mathrm{mg}, 10.8 \%(n=4)$; $400 \mathrm{mg}, 59.5 \%(n=22) ; 600 \mathrm{mg}, 18.9 \%(n=7)$; $800 \mathrm{mg}: 10.8 \%(n=4)$. A total of 24 patients (64.9\%) discontinued sorafenib treatment. Reasons for discontinuation were disease progression $(n=22)$ and AEs $(n=2)$; hepatic dysfunction $(n=1)$ and palpitation $(n=1)$. The percentage of patients with a maintenance daily dose of sorafenib $\geq 400 \mathrm{mg} /$ day was $89.2 \%$.

Drug-related AEs were found in all patients. The most common drug-related AEs were hand-foot syndrome $(32.4 \%)$ followed by diarrhea $(16.2 \%)$ and malaise $(13.5 \%)$. Two patients 


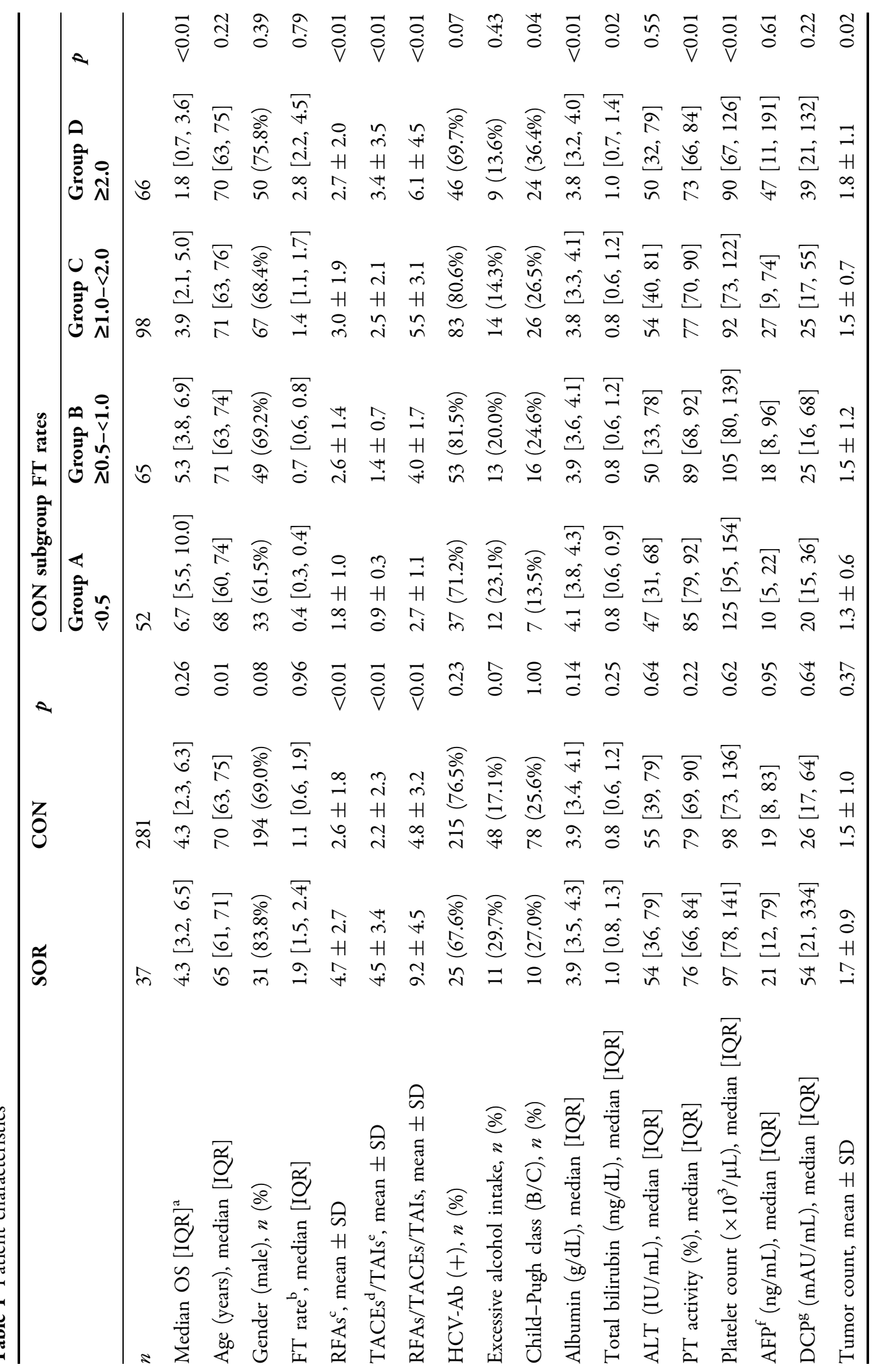



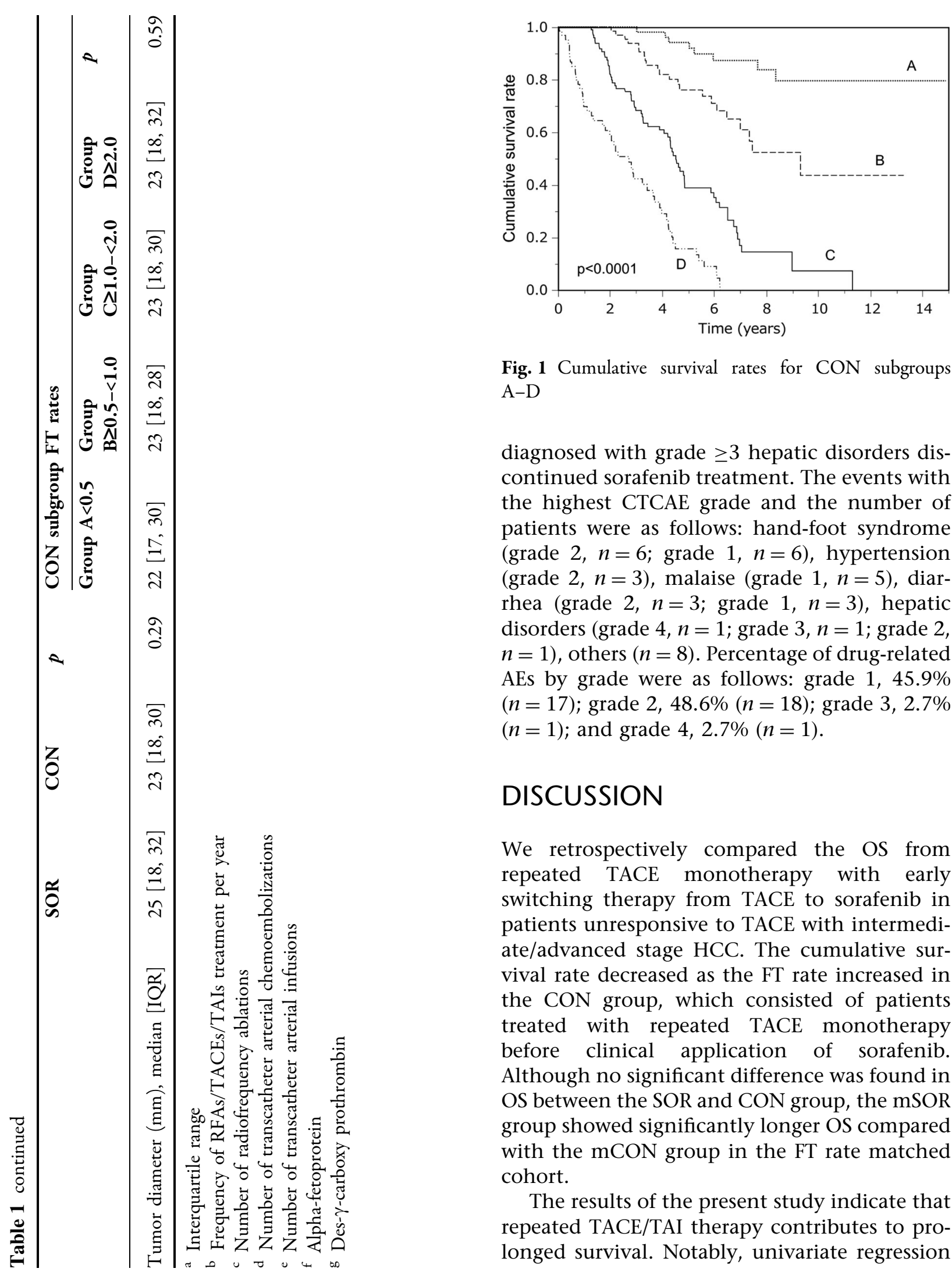

Fig. 1 Cumulative survival rates for CON subgroups A-D

diagnosed with grade $\geq 3$ hepatic disorders discontinued sorafenib treatment. The events with the highest CTCAE grade and the number of patients were as follows: hand-foot syndrome (grade 2, $n=6$; grade 1, $n=6$ ), hypertension (grade $2, n=3$ ), malaise (grade $1, n=5$ ), diarrhea (grade $2, n=3$; grade $1, n=3$ ), hepatic disorders (grade $4, n=1$; grade $3, n=1$; grade 2, $n=1)$, others $(n=8)$. Percentage of drug-related AEs by grade were as follows: grade 1, 45.9\% $(n=17)$; grade $2,48.6 \%(n=18)$; grade $3,2.7 \%$ $(n=1)$; and grade $4,2.7 \%(n=1)$.

\section{DISCUSSION}

We retrospectively compared the OS from repeated TACE monotherapy with early switching therapy from TACE to sorafenib in patients unresponsive to TACE with intermediate/advanced stage HCC. The cumulative survival rate decreased as the FT rate increased in the CON group, which consisted of patients treated with repeated TACE monotherapy before clinical application of sorafenib. Although no significant difference was found in OS between the SOR and CON group, the mSOR group showed significantly longer OS compared with the mCON group in the FT rate matched cohort.

The results of the present study indicate that repeated TACE/TAI therapy contributes to prolonged survival. Notably, univariate regression 
Table 2 Factors influencing survival in CON group

\begin{tabular}{|c|c|c|c|c|}
\hline \multirow[t]{2}{*}{ Variables } & \multicolumn{4}{|c|}{ Hazard ratio $[95 \% \mathrm{CI}]$} \\
\hline & Unadjusted & $p$ & Adjusted & $p$ \\
\hline Age (/years old $)$ & $1.01[0.99-1.03]$ & 0.25 & - & \\
\hline Gender (male) & $1.10[0.78-1.55]$ & 0.58 & - & \\
\hline $\operatorname{RFAs}^{\mathrm{a}}$ (/procedure) & $0.79[0.70-0.88]$ & $<0.01$ & $0.56[0.49-0.64]$ & $<0.01$ \\
\hline $\mathrm{TACEs}^{\mathrm{b}} / \mathrm{TAIs}^{\mathrm{c}}$ (/procedure) & $1.07[1.02-1.12]$ & 0.02 & $0.85[0.78-0.92]$ & $<0.01$ \\
\hline FT $_{\text {rate }}{ }^{\mathrm{d}} \geq 1.0$ & $6.85[4.50-10.4]$ & $<0.01$ & $30.3[16.7-55.6]$ & $<0.01$ \\
\hline $\mathrm{HCV}-\mathrm{Ab}(+)$ & $1.33[0.88-2.01]$ & 0.75 & - & \\
\hline $\operatorname{Albumin}(/ 1.0 \mathrm{~g} / \mathrm{dL})$ & $0.45[0.34-0.59]$ & $<0.01$ & $0.71[0.49-1.05]$ & 0.08 \\
\hline Total bilirubin $(/ 1.0 \mathrm{mg} / \mathrm{dL})$ & $1.82[1.41-2.36]$ & $<0.01$ & $1.33[0.92-1.93]$ & 0.13 \\
\hline $\operatorname{ALT}(/ 10 \mathrm{IU} / \mathrm{mL})$ & $1.00[0.99-1.02]$ & 0.21 & - & \\
\hline PT activity $(/ 1 \%)$ & $0.98[0.97-0.99]$ & $<0.01$ & $0.99[0.97-1.00]$ & 0.11 \\
\hline Platelet count $\left(/ 10^{3} / \mu \mathrm{L}\right)$ & $0.93[0.90-0.97]$ & $<0.01$ & $1.01[0.98-1.04]$ & 0.40 \\
\hline $\operatorname{AFP}^{\mathrm{e}}(/ 10 \mathrm{ng} / \mathrm{mL})$ & $1.01[1.00-1.03]$ & 0.02 & $1.00[0.99-1.01]$ & 0.42 \\
\hline $\operatorname{DCP}^{\mathrm{f}}(/ 10 \mathrm{mAU} / \mathrm{mL})$ & $1.01[1.00-1.03]$ & 0.02 & $1.00[0.99-1.01]$ & 0.46 \\
\hline Tumor count (/1 tumor) & $1.10[0.98-1.24]$ & 0.11 & - & \\
\hline Tumor diameter $(/ 1 \mathrm{~mm})$ & $1.00[0.98-1.02]$ & 0.84 & - & \\
\hline
\end{tabular}

${ }^{a}$ Radiofrequency ablation

b Transcatheter arterial chemoembolization

c Transcatheter arterial infusion

d Frequency of RFAs/TACEs/TAIs treatment per year

e Alpha-fetoprotein

${ }^{f}$ Des- $\gamma$-carboxy prothrombin

analysis showed that repeated TACE/TAI therapy was a significant poor prognostic factor. However, multivariate analysis showed that it significantly contributed to better survival, indicating that there were two types of clinical courses in patients with repeated TACE/TAI therapy. One is repeated TACE/TAI under poor response to the therapy due to the rapid progress of HCC; the other is effectively repeated TACE/TAI to control the condition of the disease. Multivariate analysis of the adjusted influential factors including FT rate revealed the efficacy of TACE/TAI on survival, indicating the difficulty of evaluating HCC treatment. These results can be interpreted to conclude that TACE/TAI is an effective therapy if delivered to appropriate cases.
The SOR group had received more RFA and TACE/TAI procedures and showed a higher FT rate compared with the CON group. The FT rate of the SOR group compared with CON subgroups shows that it lies midway between the $\mathrm{C}$ and $\mathrm{D}$ groups (Table 1). Median survival of the SOR group was longer than that of the C group, even though the FT rate of the SOR group was higher than that in $\mathrm{C}$ group, indicating that sorafenib might contribute to maintain a condition acceptable for receiving treatment in patients with intermediate/advanced stage HCC. The FT rate was strongly associated with the survival, and the HR (95\% CI) for death was $30.3(16.7-55.6)$ in patients with an FT rate of $\geq 1.0$. The survival was affected by various conditions including tumor progression, 
Table 3 Patient characteristics in FT rate matched cohort

\begin{tabular}{|c|c|c|c|}
\hline & $\operatorname{mSOR}(n=35)$ & $\operatorname{mCON}(n=35)$ & $p$ \\
\hline Age (years old), median $[\mathrm{IQR}]^{\mathrm{a}}$ & $65.5[61.7,71.5]$ & $68.1[59.3,75.3]$ & 0.47 \\
\hline Gender (male), $n(\%)$ & $29(82.9 \%)$ & $21(60.0 \%)$ & 0.034 \\
\hline FT rate $^{\mathrm{b}}$, median $[\mathrm{IQR}]$ & $1.90[1.49,2.30]$ & $1.91[1.48,2.30]$ & 0.99 \\
\hline $\mathrm{RFAs}^{\mathrm{c}}$, mean $\pm \mathrm{SD}$ & $4.74 \pm 2.72$ & $2.83 \pm 1.60$ & $<0.001$ \\
\hline TACEs $^{\mathrm{d}} /$ TAIs $^{\mathrm{e}}$, mean \pm SD & $3.37 \pm 2.99$ & $2.34 \pm 2.88$ & 0.14 \\
\hline RFAs/TACEs/TAIs, mean \pm SD & $8.80 \pm 4.37$ & $5.97 \pm 3.71$ & 0.004 \\
\hline $\mathrm{HCV}+, n(\%)$ & $24(68.6 \%)$ & $28(80.0 \%)$ & 0.27 \\
\hline Child-Pugh class (B/C), $n(\%)$ & $10(28.6 \%)$ & $14(40.0 \%)$ & 0.31 \\
\hline Albumin $(\mathrm{g} / \mathrm{dL})$, median $[\mathrm{IQR}]$ & $3.8[3.4,4.2]$ & $3.6[3.2,3.9]$ & 0.016 \\
\hline Total-bilirubin (mg/dL), median [IQR]) & $1.0[0.7,1.3]$ & $1.0[0.7,1.5]$ & 0.57 \\
\hline $\operatorname{ALT}(\mathrm{IU} / \mathrm{mL})$, median $[\mathrm{IQR}]$ & $54[36,79]$ & $54[34,96]$ & 0.80 \\
\hline PT activity (\%), median [IQR] & $74.3[65.7,80.6]$ & $71.2[64.3,76.9]$ & 0.54 \\
\hline Platelet count $\left(\times 10^{3} / \mu \mathrm{L}\right)$, median $[\mathrm{IQR}]$ & $9.7[7.3,14.1]$ & $8.3[6.5,10.6]$ & 0.094 \\
\hline $\operatorname{AFP}^{f}(n g / m L)$, median [IQR] & $17.0[8.0,78.5]$ & $73.4[18.4,256.0]$ & 0.030 \\
\hline $\mathrm{DCP}^{\mathrm{g}}(\mathrm{mAU} / \mathrm{mL})$, median [IQR] & $33[21,292]$ & $38[22,132]$ & 0.98 \\
\hline Tumor count, mean $\pm S D$ & $1.68 \pm 0.90$ & $1.86 \pm 1.12$ & 0.48 \\
\hline Tumor diameter $(\mathrm{mm})$, mean $\pm S D$ & $25.9 \pm 9.4$ & $26.9 \pm 11.7$ & 0.67 \\
\hline
\end{tabular}

a Interquartile range

b Frequency of RFAs/TACEs/TAIs treatment per year

c Number of radiofrequency ablation

d Number of transcatheter arterial chemoembolization

e Number of transcatheter arterial infusion

f Alpha-fetoprotein

g Des- $\gamma$-carboxy prothrombin

malignancy grade, and therapeutic situation. We consider the FT rate to be an index that reflects these conditions. The evaluation of the FT rate matched cohort shows the efficacy of sorafenib therapy on OS as a result of adjustment of these factors. Kudo et al. reported that there was no difference in OS between sorafenib and placebo in 458 unresectable HCC patients who responded to TACE [15]. In addition, Lencioni et al. reported that there was no difference in OS between treatment with sorafenib or placebo plus TACE with doxorubicin-eluting beads in 307 intermediate stage HCC patients [16]. On the other hand, Bai et al. reported that sorafenib plus TACE therapy significantly prolonged OS compared with TACE monotherapy in unresectable HCC patients in the cohort with propensity scores matched for age, gender, hepatitis virus, tumor metastasis, treatment history, BCLC stage, Child-Pugh class, ECOG performance status, and ascites [26]. Our findings indicate that various factors, other than the therapy drugs, strongly affected outcome including OS in patients with HCC. Considering the above, the FT rate may be useful in evaluating the outcome of specific treatments in a population of patients at various stages and receiving different treatments. 

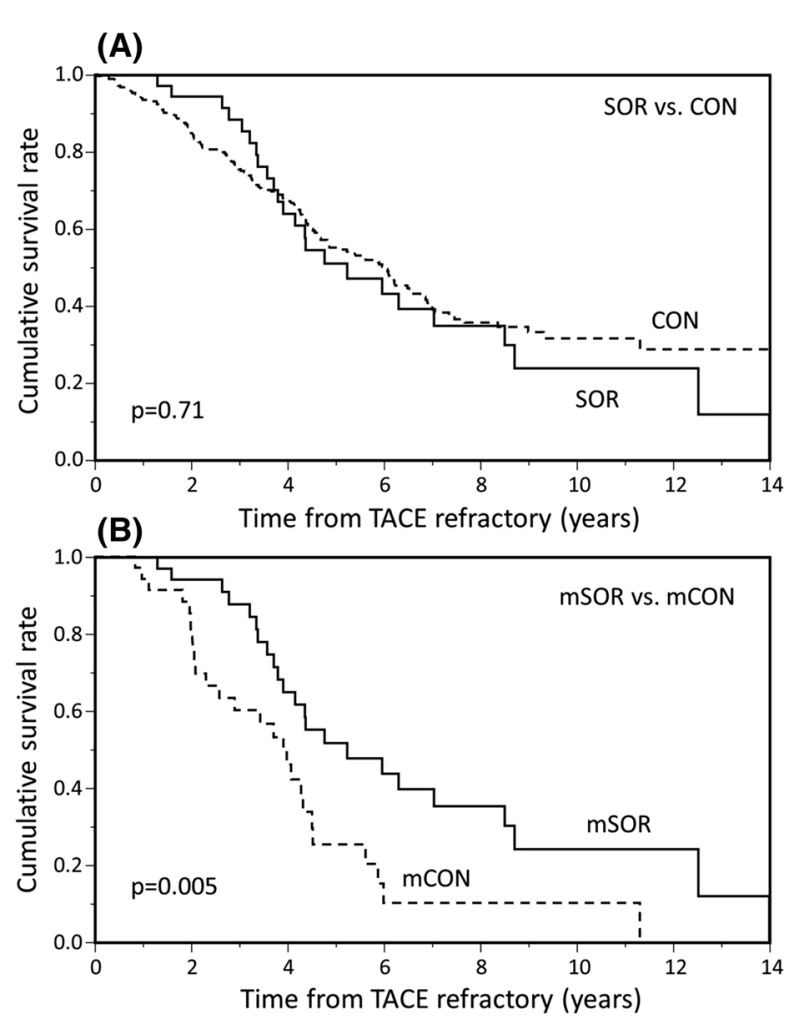

Fig. 2 Cumulative survival rates for a SOR vs. CON and b $\mathrm{mSOR}$ vs. $\mathrm{mCON}$
In the FT rate matched cohort, the numbers of RFA and RFA/TACE/TAI treatments were significantly higher in the mSOR group compared with the mCON group. In theory, FT rates being equal, more TACE/TAI procedures correspond to a longer survival period. The numbers of TACE/TAI treatments were higher in the SOR group compared with the CON group. Reported side effects of TACE are increased vascular endothelial growth factor (VEGF) expression and increased possibility of the recurrence of HCC [27]. Sorafenib inhibits neoangiogenesis by blocking VEGF receptors [10]. Zhang et al. reported the possibility that sorafenib reduces the side effects of TACE via pharmacological action thorough the VEGF receptor 2 pathway [28]. These findings indicate that sorafenib suppresses tumor growth and maintains a condition that can be treated by TACE/TAI.

However, both Kudo et al. and Lencioni et al. reported that sorafenib did not contribute to OS from maintenance therapy after TACE or combination therapy with TACE. In Kudo's study, sorafenib was started at 9 weeks or later after TACE in over $50 \%$ of patients and sorafenibtreated patients required dose reductions (73\%) and/or interruptions (91\%) [15]. In Lencioni's

Table 4 Factors influencing survival in FT rate matched cohort

\begin{tabular}{|c|c|c|c|c|}
\hline \multirow[t]{2}{*}{ Variables } & \multicolumn{4}{|c|}{ Hazard ratio $[95 \% \mathrm{CI}]$} \\
\hline & Unadjusted & $p$ & Adjusted & $p$ \\
\hline Gender (male) & $0.46[0.25-0.8]$ & 0.012 & $0.96[0.47-2.00]$ & 0.90 \\
\hline Age (/years old $)$ & $1.04[1.00-1.0]$ & 0.037 & $1.03[0.99-1.0]$ & 0.14 \\
\hline Sorafenib & $0.44[0.24-0.8]$ & 0.006 & $0.42[0.22-0.8]$ & 0.008 \\
\hline $\mathrm{FT} \mathrm{rate}^{\mathrm{a}}(/ 1.0)$ & $3.34[1.95-5.8]$ & $<0.001$ & $2.86[1.61-5.2]$ & $<0.001$ \\
\hline Tumor diameter $(/ 1 \mathrm{~mm})$ & $1.00[0.97-1.0]$ & 0.83 & - & - \\
\hline Tumor count (/1 tumor) & $1.08[0.79-1.4]$ & 0.59 & - & - \\
\hline Total bilirubin $(/ 1 \mathrm{mg} / \mathrm{dL})$ & $1.19[0.65-2.0]$ & 0.55 & - & - \\
\hline PT activity $(/ 1 \%)$ & $0.98[0.96-1.0]$ & 0.058 & - & - \\
\hline Albumin $(/ 1 \mathrm{~g} / \mathrm{dL})$ & $0.14[0.99-1.0]$ & 0.076 & - & - \\
\hline $\mathrm{AFP}^{\mathrm{b}}(/ 1 \mathrm{ng} / \mathrm{mL})$ & $0.61[0.36-1.0]$ & 0.061 & - & - \\
\hline Platelet count $\left(/ 10^{3} / \mu \mathrm{L}\right)$ & $0.92[0.87-0.9]$ & 0.011 & $0.95[0.89-1.0]$ & 0.10 \\
\hline
\end{tabular}

a Frequency of RFAs/TACEs/TAIs treatment per year

b Alpha-fetoprotein 
study, the median daily dose of sorafenib was approximately $70 \%$ of the planned dose and AEs were the principal reason for dose modification [16]. In the present study, although AEs were found in all 37 patients treated with sorafenib, only two patients discontinued sorafenib as a result of AEs. The median treatment duration period of sorafenib was 373 days, with a maintenance of $400 \mathrm{mg} /$ day or more in $89.2 \%$ of the patients. Hiramine et al. reported that the survival rate in patients with unresectable HCC patients significantly declined in cases with a sorafenib duration period of less than 60 days [29]. Although continuous administration of sorafenib improves the survival in HCC patients, AEs might obstruct its duration. The results from analysis of the FT rate matched cohort in this study show that sorafenib treatment significantly contributed to OS with an HR $(95 \% \mathrm{CI})$ of $0.42(0.22-0.80)$. The duration of the treatment period and dosing of sorafenib might be one of the reasons for this result.

This study has a several limitations. First, this is a retrospective analysis and thus it is impossible to eliminate all bias and confounding factors. Second, the FT rate does not reflect the treatment interval, which may fluctuate with state of disease. Third, the FT rate was determined using the total number of treatments of RFA, which is a curative treatment applied to early stage HCC, and TACE, which is not a curative treatment applied to intermediate stage HCC. Fourth, the term of survival was calculated with HCC diagnosis as the starting point and was not calculated from diagnosis to loss of response to TACE. Fifth, the propensity score was calculated using only the FT rate, without considering other factors. Therefore, this study should be interpreted as the proof of concept for the FT rate.

\section{CONCLUSION}

For those patients not expected to have longterm survival from repeated TACE monotherapy, early switching to sorafenib from TACE may prolong OS. The present study indicates that the FT rate is a useful index in evaluating the outcome in patients at various stages of disease and receiving various treatment regimens.

\section{ACKNOWLEDGEMENTS}

Sponsorship for this study, article processing charges, and the open access fee were funded by Bayer Yakuhin, Ltd. The authors wish to thank Springer Healthcare Communications and Tetsuhiko Yokoyama for providing medical writing support, which was funded by Bayer Yakuhin, Ltd. All authors had full access to all of the data in this study and take complete responsibility for the integrity of the data and accuracy of the data analysis. All named authors meet the International Committee of Medical Journal Editors (ICMJE) criteria for authorship for this manuscript, take responsibility for the integrity of the work as a whole, and have given final approval for the version to be published.

Disclosures. Takamasa Ohki has received lecture fees from Bayer Yakuhin, Ltd, Otsuka Pharmaceutical Co., Ltd, Sumitomo Dainippon Pharma Co., Ltd, Shionogi \& Co., Ltd., Gilead Sciences, Inc, AbbVie Inc, and EA Pharma Co., Ltd. Mayuko Kondo, Yuki Karasawa, Satoshi Kawamura, Shuuya Maeshima, Kentaro Kojima, Michiharu Seki, Nobuo Toda, Yoshinobu Shioda, and Kazumi Tagawa declare that they have no conflict of interest. Article processing charges and open access fee were funded by Bayer Yakuhin, Ltd.

Compliance with Ethics Guidelines. All procedures followed were in accordance with the ethical standards of the responsible committee on human experimentation (institutional and national) and with the 2013 revised Helsinki Declaration of 1964. Informed consent was obtained from all patients included in the study.

Data Availability. The datasets during and/ or analyzed during the current study are available from the corresponding author on reasonable request. 
Open Access. This article is distributed under the terms of the Creative Commons Attribution-NonCommercial 4.0 International License (http://creativecommons.org/licenses/ by-nc/4.0/), which permits any noncommercial use, distribution, and reproduction in any medium, provided you give appropriate credit to the original author(s) and the source, provide a link to the Creative Commons license, and indicate if changes were made.

\section{REFERENCES}

1. GLOBOCAN 2012 v1.0. Estimated cancer incidence, mortality, and prevalence worldwide. http:// globocan.iarc.fr. Accessed 13 Jan 2017.

2. Tokushige K, Hyogo H, Nakajima T, et al. Hepatocellular carcinoma in Japanese patients with nonalcoholic fatty liver disease and alcoholic liver disease: multicenter survey. J Gastroenterol. 2016;51:586-96.

3. Valenzuela A, Ha NB, Gallo A, et al. Recurrent hepatocellular carcinoma and poorer overall survival in patients undergoing left-sided compared with right-sided partial hepatectomy. J Clin Gastroenterol. 2015;49:158-64.

4. Morise Z, Kawabe N, Tomishige H, et al. Recent advances in the surgical treatment of hepatocellular carcinoma. World J Gastroenterol. 2014;20:14381-92.

5. Reig M, Darnell A, Forner A, Rimola J, Ayuso C, Bruix J. Systemic therapy for hepatocellular carcinoma: the issue of treatment stage migration and registration of progression using the BCLC-refined RECIST. Semin Liv Dis. 2014;34:444-55.

6. Cheng HY, Wang X, Chen D, Xu AM, Jia YC. The value and limitation of transcatheter arterial chemoembolization in preventing recurrence of resected hepatocellular carcinoma. World J Gastroenterol. 2005;11:3644-6.

7. Llovet JM, Real MI, Montana X, et al. Arterial embolization or chemoembolization versus symptomatic treatment in patients with unresectable hepatocellular carcinoma: a randomized controlled trial. Lancet. 2002;359:1734-9.

8. Lo CM, Ngan H, Tso WK, et al. Randomized controlled trial of transarterial lipiodol chemoembolization for unresectable hepatocellular carcinoma. Hepatology. 2002;35:1164-71.
9. Kudo M, Izumi N, Kokudo N, et al. Management of hepatocellular carcinoma in Japan: consensus-based clinical practice guidelines proposed by the Japan Society of Hepatology (JSH) 2010 updated version. Dig Dis. 2011;29:339-64.

10. Wilhelm SM, Carter C, Tang L, et al. BAY 43-9006 exhibits broad spectrum oral antitumor activity and targets the RAF/MEK/ERK pathway and receptor tyrosine kinases involved in tumor progression and angiogenesis. Cancer Res. 2004;64:7099-109.

11. Llovet JM, Ricci S, Mazzaferro V, et al. Sorafenib in advanced hepatocellular carcinoma. N Engl J Med. 2008;359:378-90.

12. Cheng AL, Kang YK, Chen Z, et al. Efficacy and safety of sorafenib in patients in the Asia-Pacific region with advanced hepatocellular carcinoma: a phase III randomized, double-blind, placebo-controlled trial. Lancet Oncol. 2009;10:25-34.

13. Ogasawara S, Chiba T, Ooka Y, et al. Efficacy of sorafenib in intermediate-stage hepatocellular carcinoma patients refractory to transarterial chemoembolization. Oncology. 2014;87:330-41.

14. Arizumi T, Ueshima K, Minami T, et al. Effectiveness of sorafenib in patients with transcatheter arterial chemoembolization (TACE) refractory and intermediate-stage hepatocellular carcinoma. Liv Cancer. 2015;4:253-62.

15. Kudo M, Imanaka $\mathrm{K}$, Chida N, et al. Phase III study of sorafenib after transarterial chemoembolization in Japanese and Korean patients with unresectable hepatocellular carcinoma. Eur J Cancer. 2011;47:2117.

16. Lencioni R, Llovet JM, Han G, et al. Sorafenib or placebo plus TACE with doxorubicin-eluting beads for intermediate stage HCC: the SPACE trial. J Hepatol. 2016;64:1090-8.

17. Zeng J, Lv L, Mei ZC. Efficacy and safety of transarterial chemoembolization plus sorafenib for early or intermediate stage hepatocellular carcinoma: a systematic review and meta-analysis of randomized controlled trials. Clin Res Hepatol Gastroenterol. 2016;40:688-97.

18. Hu MD, Jia LH, Liu HB, Zhang KH, Guo GH. Sorafenib in combination with transarterial chemoembolization for hepatocellular carcinoma: a meta-analysis. Eur Rev Med Pharmacol Sci. 2016;20:64-74.

19. Yang M, Yuan JQ, Bai M, Han GH. Transarterial chemoembolization combined with sorafenib for unresectable hepatocellular carcinoma: a systematic review and meta-analysis. Mol Biol Rep. 2014;41:6575-82. 
20. Zhang L, Hu P, Chen X, Bie P. Transarterial chemoembolization (TACE) plus sorafenib versus TACE for intermediate or advanced stage hepatocellular carcinoma: a meta-analysis. PLoS One. 2014;9:e100305.

21. Fu QH, Zhang Q, Bai XL, et al. Sorafenib enhances effects of transarterial chemoembolization for hepatocellular carcinoma: a systematic review and meta-analysis. J Cancer Res Clin Oncol. 2014;140:1429-40.

22. Liu L, Chen H, Wang M, et al. Combination therapy of sorafenib and TACE for unresectable HCC: a systematic review and meta-analysis. PLoS One. 2014;9:e91124.

23. Ohki T, Sato K, Yamagami M, et al. Efficacy of transcatheter arterial chemoembolization followed by sorafenib for intermediate/advanced hepatocellular carcinoma in patients in Japan: a retrospective analysis. Clin Drug Investig. 2015;35:751-9.

24. Ohki T, Sato K, Yamagami $M$, et al. Erratum to: Efficacy of transcatheter arterial chemoembolization followed by sorafenib for intermediate/advanced hepatocellular carcinoma in patients in Japan: a retrospective analysis. Clin Drug Investig. 2016;36:93-6.
25. Lencioni R, Llovet JM. Modified RECIST (mRECIST) assessment for hepatocellular carcinoma. Semin Liv Dis. 2010;30:52-60.

26. Bai W, Wang YJ, Zhao Y, et al. Sorafenib in combination with transarterial chemoembolization improves the survival of patients with unresectable hepatocellular carcinoma: a propensity score matching study. J Dig Dis. 2013;14:181-90.

27. Li X, Feng GS, Zheng CS, et al. Expression of plasma vascular endothelial growth factor in patients with hepatocellular carcinoma and effect of transcatheter arterial chemoembolization therapy on plasma vascular endothelial growth factor level. World J Gastroenterol. 2004;10(19):2878-82.

28. Zhang $\mathrm{X}$, Wang $\mathrm{K}$, Wang $\mathrm{M}$, et al. Transarterial chemoembolization (TACE) combined with sorafenib versus TACE for hepatocellular carcinoma with portal vein tumor thrombus: a systematic review and meta-analysis. Oncotarget. 2017. doi:10. 18632/oncotarget. 15075 .

29. Hiramine $Y$, Uto H, Imamura $Y$, et al. Sorafenib and hepatic arterial infusion chemotherapy for unresectable advanced hepatocellular carcinoma: a comparative study. Exp Ther Med. 2011;2:433-41. 